\section{ANTIMICROBIAL RESISTANCE OF STREPTOCOCCUS PNEUMONIAE ISOLATED FROM CHILDREN WITH NASOPHARYNGEAL CARRIAGE AND INFECTIONS DURING THE 9-YEAR PERIOD (1999-2007)}

\author{
K. Semczuk, A. Pawinska, D. Borowiec, \\ B. Garczewska, W. Kamińska, \\ K. Dzierżanowska-Fangrat
}

\section{Clinical Microbiology, The Children's Memorial Heath Institute, Warsaw, Poland}

Aim: Streptococcus pneumoniae continues to be an important cause of community-acquired respiratory tract infections. Because of increase in its antimicrobial resistance, the surveillance of the local antimicrobial susceptibility of S.pneumoniae should be carried out regularly.

Methods: Streptococcus pneumoniae strains were isolated from children on admission to The Children's Memorial Health Institute, Warsaw, Poland between Jan 1999 and Dec 2007. A total of 784 isolates of S.pneumoniae were collected. Susceptibility to antibiotics was analyzed in two periods: 1999-2002 and 2003-2007. All the strains (185) collected in the first period originated from children with pneumococcal diseases. Of the 599 strains obtained in the second period, 234 were isolated from children with S.pneumoniae infection and 365 from children with nasopharyngeal carriage.

Results and conclusions: Rate of resistance to penicillin was stable during the whole period of the study (3\% vs. $4 \%)$. Higher resistance rates to erythromycin (19\% vs. $29 \%)$, clindamycin (14\% vs. $26 \%$ ) and lower resistance rates to tetracycline ( $37 \%$ vs. $9 \%)$ and co-trimoxazole ( $47 \%$ vs. $41 \%)$ were observed among isolates obtained in the second period. S.pneumoniae strains isolated from infections and from carriage exhibited similar resistance patterns.

\section{PSYCHOSCOIAL BARRIERS TO ADHERENCE OF SUBSIDISED ART IN CHILDREN \& ADOLESCENTS, KAMPALA, UGANDA}

B. Freyne ${ }^{1,2}$, J. Earnest ${ }^{2}$, V. Musiime ${ }^{1}$, F. Ssali ${ }^{1}$, P. Mugyenyi ${ }^{1}$

${ }^{1}$ Paediatric Department, Joint Clinical Reseach Centre, Kampala, Uganda, ${ }^{2}$ Centre for International Health, Curtin University of Technology, Perth, WA, Australia

The aim of this study was to establish poor adherence as the primary reason for treatment failure in HIV+ children receiving subsidized ART in Uganda. The study examined psychological, cultural and structural barriers to adherence which are of increasing relevance as HIV transitions to a chronic disease of childhood in this population.

This study was exploratory and cross sectional in nature. Quantitative data was obtained from 108 charts of patients who were receiving $2^{\text {nd }}$ line ART regimens to assess clinical parameters relating to treatment failure and viral resistance. Qualitative data was obtained from 35 semi-structured interviews, four focus group discussions and four key informant interviews with caregivers of patients and staff and was analyzed using the deductive framework approach.

Quantitative data confirmed that patients were frequently switched to more costly $2^{\text {nd }}$ line regimens due to poor adherence. The mean time to development of resistance on treatment was 2.5 years. Qualitative data outlined multiple culturally specific barriers to adherence with psychosocial factors such as stigma, disclosure and family dynamics emerging as strongly as structural factors such as medication tolerability, poverty and access. Vulnerable groups warranting comprehensive treatment. packages were identified.

This study outlines the major issues facing program planners as they enter the next phase of universal access to ART in resource limited settings. Improved adherence over time is essential to effective patient care as well as program sustainability. The psychosocial issues surrounding the care of patients with HIV are inadequately catered for and are pivotal in ensuring ART adherence. 